\title{
STEM Observations of Twisting in Colloidal CdSe Quantum Rods.
}

\author{
Zhiheng Yu* , Megan Hahn ${ }^{* *}$, Todd Krauss ${ }^{* *}$ and John Silcox ${ }^{* * *}$ \\ * Physics Department, Cornell University, Ithaca, NY 14853 \\ ** Chemistry Department, University of Rochester, Rochester, NY 14627 \\ *** School of Applied and Engineering Physics, Cornell University, Ithaca, NY 14853
}

For nanoparticles with thicknesses of a few nanometers, a tiny electron probe can be used to obtain high-resolution ADF images and localized CBED patterns to reveal local crystal structure and information of orientation. In this paper, we report investigations of the local structure of colloidal $\mathrm{CdSe}$ quantum rods from high-resolution ADF images and nanodiffraction patterns by operating a STEM under different optical conditions.

Colloidal CdSe nanoparticles are a novel optical and electronic material and have shown potential for a wide range of applications ${ }^{1}$. CdSe quantum rods were synthesized according to modified versions of the methods reported in the literature ${ }^{2}$ and were stored in hexane. The electron microscopy specimens were made by depositing a $\sim 20$ microliter drop of the hexane solution onto a holey carbon film covered copper grid. The experiments were done on the Cornell VG HB501 100kV UHV STEM, which can be operated under different optical settings. Under high-resolution conditions, with one condenser lens excited, a small probe of $2 \AA$ FWHM size with a convergent angle of $10 \mathrm{mrad}$ is generated. With two condenser lenses energized at a specific setting, a broad probe of $\sim 20 \AA$ FWHM size with a smaller convergence angle (a couple of mrads) is generated. Localized nanodiffraction ${ }^{3}$ patterns can be obtained by focusing the broad probe on certain places of the sample. The main advantage of using the broad probe for localized CBED pattern determination is that radiation damage is lower since the beam is spread over a broader region on the specimen. Since the broad probe has a much smaller convergent angle, the diffraction disks are much smaller and the resultant CBED patterns (without overlapping between neighboring disks as in the case for the small probe) are much simpler to interpret in terms of the local crystal structure.

Figure 1 shows the high-resolution images and nanodiffraction patterns from various places on a colloidal CdSe quantum rod. On the left is a low magnification image to show the overall shape and size of a typical quantum rod. The central column shows the high-resolution images obtained with the small probe from various places of the rod indicated by the arrows. The column on the right is the localized CBED patterns obtained by the broad probe. Care was taken to make sure the corresponding diffraction patterns and the high-resolution images are from the same places on the rod when changing optical conditions. A nice two-dimensional lattice image (as shown by the top image in the central column) was obtained from the top part of the rod. The corresponding diffraction pattern (the top one in the column on the right) confirms that the top part of the quantum rod has the wurtzite structure that is well aligned along the $(11 \overline{2} 0)$ zone axis. The central part of the rod shows a single set of lattice fringes. The corresponding diffraction pattern indicates that this part is tilted away from the zone axis and the dominant component of the tilt is a rotation about the c-axis (the line connecting the zero disk and its strongest upper right neighbor, i. e. the $<0001>$ axis) since the diffraction disks on one side of the c-axis are stronger than that on the other side. The diffraction patterns suggest that the direction of the c-axis of the central part is the same as that of the top part although the morphology of the rod changes slightly. The bottom part of the rod is twisted in a different way as the third diffraction pattern 
suggests. A grain boundary is also detected at the "foot" of the rod, as the bottom lattice image and the bottom diffraction pattern suggest. The direction of the c-axis changes across the grain boundary. ${ }^{4}$

\section{References:}

\section{A. Malko et al., Applied Physics Letters 81 (2002) 1303.}

2. X. Peng, L. Manna, W. Yang, J. Wickham, E. Scher, A. Kadavanich, and A. P. Alivisatos, Nature 404, 59, (2000); L. Manna, E. C. Scher, and A. P. Alivisatos, J. Am. Chem. Soc. 122, 12700, (2000); Z. A. Peng, and X. Peng, J. Am. Chem. Soc. 124,3343, (2002).

3. J. M. Cowley, F.A. Sundell, Ultramicroscopy 68, 1 (1997).

4. This work was supported by the Center for Nanoscale Systems (CNS), through the Nanoscale Science and Engineering Initiative of the National Science Foundation (EEC-0117770), the Center for Future Health at the University of Rochester and the Rochester Infotonics Center of Excellence.



Figure 1. ADF-STEM images and nanodiffraction patterns from a colloidal CdSe quantum rod. On the left is a low magnification image. In the center are the high-resolution lattice images obtained with the $2 \AA$ probe from various places of the rod. On the right are nanodiffraction patterns obtained with the $20 \AA$ probe. 